\title{
A Procedure for Conformal Mapping of Triply-Connected Domains
}

\section{By Stefan Bergman and Bruce Chalmers*}

1. Introduction. The theory of the kernel function permits us to introduce the invariant metric given by

$$
d s_{\Omega}(z)=\left[K_{\Omega}(z, \bar{z})\left(d x^{2}+d y^{2}\right)\right]^{1 / 2}, \quad z=x+i y, \quad \bar{z}=x-i y,
$$

and the absolute invariant

$$
J_{\Omega}(z) \equiv J_{\Omega}(z, \bar{z})=\frac{1}{K_{\Omega}} \frac{\partial^{2} \log K_{\Omega}}{\partial z \partial \bar{z}}, \quad K_{\Omega} \equiv K_{\Omega}(z, \bar{z}) .
$$

Here $\Omega$ is a domain in the $z$-plane. Thus (1) and (2) are domain functionals possessing the following property: let $\Omega$ (in the $z$-plane) and $B$ (in the $Z$-plane) be two domains which can be transformed by the CT (conformal transformation)

$$
W: Z=Z(z)
$$

onto each other. Then

$$
d s_{\Omega}(z)=d s_{B}(Z), \quad J_{\Omega}(z, \bar{z})=J_{B}\left(Z(z),\left[\overline{Z(z)}^{1 / 2}\right)\right.
$$

(see [B.5, p. 36 (25)], [B.6], [B.8], [B.9], and also [E.1], [F.1], and [M.1]).

In the following we shall assume that $\partial B$ consists of $n$ twice continuously differentiable closed curves $\beta_{\kappa}, \kappa=1,2, \cdots, n, 1 \leqq n<\infty$, and the radius of curvature of every $\beta_{\kappa}$ is uniformly bounded.

One can show that $J_{B}(Z, \bar{Z})$ has the boundary value $2 \pi$. More exactly, let $\left(Z_{\nu}\right)$, $\nu=1,2, \cdots$, be a set of points converging to $Q, Q \in \partial B$, and lying in the angular domain whose boundary lines form the angle $\alpha$ with the interior normal at $Q$, $|\alpha|<\pi / 2$.

Then

$$
\lim _{Z_{n} \rightarrow Q} J_{B}\left(Z_{n}, \bar{Z}_{n}\right)=2 \pi
$$

[B.5, p. 39].

If $B$ is a simply connected domain, i.e., $n=1$, then

$$
J_{B}(Z, \bar{Z})=\text { const }=2 \pi, \quad Z \in B .
$$

In the case of $n$-ply connected domains, $2 \leqq n<\infty, J_{B}(Z, \bar{Z})$ (in general) is not constant. It can be used to determine whether two domains, say $\Omega$ (in the $z$-plane) and $B$ (in the $Z$-plane), can be mapped conformally onto each other and, if this is the case, to construct the mapping function.

Remark. The theory of the kernel function can be used in different ways for the numerical computation of CT's of multiply-connected domains onto canonical do-

Received February 3, 1967.

* This work was supported in part by Air Force contract AF-1047-66 at Stanford University. 
mains. The procedure considered in the present paper is different from that described in [5, Chapter VI]. It is of interest to study under what conditions a certain procedure is well suited for numerical purposes. This can be determined by discussing the application of the procedure and computing examples in detail.

In the case of doubly-connected domains the procedure of the present paper has been worked out in detail by Zarankiewicz [Z.1]. A generalization of Zarankiewicz's considerations to the case of $n \geqq 3$ is not immediate, and in the present paper we shall discuss the necessary modifications of the procedure in the case of $n=3$.

Every triply-connected domain can be mapped onto a domain $\Omega$ bounded by two concentric circles, from which a circular hole is cut out. Let the radii of the circles with the center at $z=0$ be $\rho_{3}$ and $\rho_{1}, \rho_{3}>\rho_{1}$, and let the radius of the third circle with the center at $z=1$ be $\rho_{2}, 2 \rho_{2}<\rho_{3}-\rho_{1}$ (see Fig. 1, p. 529). $\rho_{1}, \rho_{2}, \rho_{3}$ are the moduli of the class of domains which can be mapped conformally onto each other. Here $\rho_{3}>1$.

The moduli $\rho_{k}$ are not uniquely determined by the domain, since by a linear transformation $\Omega$ can be transformed onto a canonical domain bounded by three circles. These possibilities are discussed in Section 3 and a normalization of the mapping function is indicated, which permits us to associate the canonical domain in a unique manner.

The invariant $J_{\Omega}(z, \bar{z}) \equiv \mathcal{F}_{\Omega}(x, y)$ is an analytic function of two real variables $x, y$ in $\Omega$. Therefore in every closed subdomain $\bar{\Omega}^{\prime}$ of $\Omega\left(\bar{\Omega}^{\prime} \subset \Omega\right), J_{\Omega}$ has only a finite number of critical points. $J_{\Omega}$ assumes the boundary values $2 \pi$, see [B.5]. The level lines $J_{\Omega}=$ const are indicated in Fig. 3, p. 533 .

It should be noted that

$$
K_{B}(z, \bar{\zeta})-\iint_{\widetilde{B}} \frac{d \omega_{w}}{(z-w)^{2}(\bar{\zeta}-\bar{w})^{2}}
$$

is an analytic function of $z$ and $\bar{\zeta}$ in the closed domain $\bar{B}$. Here $\widetilde{B}$ is the complement of $B$. See [B.-S. 1, p. 214]. This result and (2) enable us to investigate the behavior of $J_{\Omega}(z, \bar{z})$ at boundary points.

A survey of results used is presented in Section 2 and the numerical computation of some tools needed for our purposes is discussed in Section 3.

In Section 4 we consider the critical points of $J_{\Omega}(z, \bar{z})$ and $J_{B}(Z, \bar{Z})$; further we discuss how it is possible, by using these quantities, to determine the moduli $\rho_{1}, \rho_{2}, \rho_{3}$ of $\Omega$, which is a conformal image of a given domain $B$.

A remark about another procedure for CT of multiply-connected domains is made in Section 5. By use of the results in Sections 3 and 4, an example of a CT mapping $B$ onto $\Omega$ is given in Section 6 .

2. A Survey of Some Results in the Theory of the Kernel Function. Let $\phi_{\nu}(z), \nu=1,2, \cdots$ represent a complete set of functions orthonormal in the domain $B$, i.e., $\phi_{\nu}(z)$ are analytic functions satisfying the relation

$$
\begin{aligned}
\iint_{B} \phi_{\nu}(z) \overline{\phi_{\mu}(z)} d \omega=\delta_{\nu \mu} & =1 \text { for } \nu=\mu, \\
& =0 \text { for } \nu \neq \mu,
\end{aligned}
$$


where $d \omega$ is the area element and $z=x+i y$. If $\phi_{\nu}(z), \nu=1,2, \cdots$, is complete for the class of square integrable functions with single-valued integrals, then

$$
K_{B}(z, \bar{z}) \equiv \sum_{\nu=1}^{\infty} \phi_{\nu}(z) \overline{\phi_{\nu}(z)}
$$

is independent of the choice of a complete set of orthonormal functions and is called the kernel function of the domain $B$ [B.5, p. 9].

The expression $d s$, see (1.1), defines a line element of a metric which is invariant with respect to CT's [B.5, p. 32]. The curvature $C_{B}(z)=-2 J_{B}(z, \bar{z})$, see (1.2), of this metric is a function which is invariant with respect to CT's [B.5, p. 36].

If we approach any boundary point $P$ of $B$, the relation

$$
\lim _{z \rightarrow P} C_{B}(z)=-4 \pi
$$

holds, provided that the boundary is twice continuously differentiable at $P$ [B.5, (41) p. 39].

$C_{B}(z)$ is a real analytic function of two real variables $x, y$ inside the domain. The critical sets of $J_{B}(z, \bar{z})$ are called interior distinguished sets. For simply-connected domains $J_{B}$ is constant in $B$. In the case of multiply-connected domains, the invariant $J_{B}$ is (in general) not constant (see Fig. 3, p. 533) and one can use its level lines and their orthogonal trajectories as a coordinate system which is invariant under CT's. Determining these lines $\left(J_{B}=\right.$ const and their orthogonal trajectories) and using the metric (1.1), one obtains a numerical procedure for carrying out a CT of a multiply-connected domain onto another, if this is possible. Concerning geodesics of metric (1.1), see [H.1].

3. Computations of Orthogonal Functions $\phi_{\nu}$ for the Canonical Domain $\Omega$. The conformal equivalence class of a domain of connectivity $n(n>2)$ is determined by $3 n-6$ real numbers which are called the moduli of the domain. Two triplyconnected domains $(n=3)$ may therefore be conformally mapped onto each other if and only if they agree in all three moduli. Hence the collection of ring domains with a circular hole, where the ring has the center at $z=0$ and the circular hole

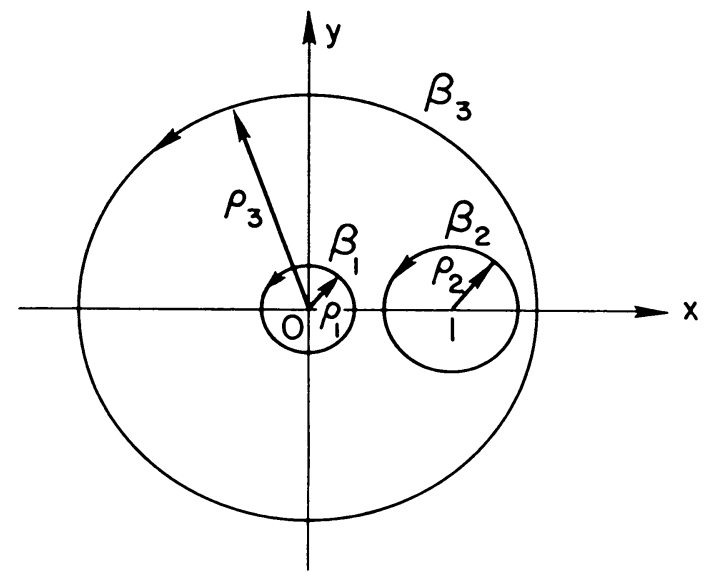

Figure 1. The domain $\Omega$. 
has the center at $z=1$, forms a complete set of canonical triply-connected domains under CT's. Evidently, one can use as the three moduli, $\rho_{1}, \rho_{2}, \rho_{3}$, the radii of the three circular boundary components $\beta_{\kappa}, \kappa=1,2,3$. See Fig. 1.

As we mentioned before, the canonical domain to a given class of conformally equivalent domains is not uniquely determined. In the case of triply-connected domains there exist six canonical domains in the class. Let us assume that $z=0$ is the center of two concentric circles $\beta_{3}$ and $\beta_{1}$ with radii $\rho_{3}$ and $\rho_{1}, \rho_{3}>\rho_{1}$, and $z=1$ is the center of the third circle $\beta_{2}$ with radius $\rho_{2}$. Then for the radii of the circles of the five other canonical domains of the same class we obtain the values

$$
\left(\rho_{1}{ }^{(2)}, \rho_{2}{ }^{(2)}, \rho_{3}{ }^{(2)}\right)=\left(\rho_{3} d, \frac{\rho_{1} \rho_{3}\left(1-a^{2}\right)}{\rho_{3}{ }^{2}+a^{2} \rho_{1}{ }^{2}}, \rho_{3}\right) \equiv S\left(\rho_{1}, \rho_{2}, \rho_{3}\right),
$$

where

$$
c=\frac{\left(\rho_{3}-\rho_{2}\right)^{2}-1}{\rho_{2} \rho_{3}}, \quad d=\frac{2+c-(c(4+c))^{1 / 2}}{2}, \quad a=\frac{\rho_{3} d-\left(1+\rho_{2}\right)}{d\left(1+\rho_{2}\right)-\rho_{3}},
$$

$$
\begin{aligned}
\left(\rho_{1}{ }^{(3)}, \rho_{2}{ }^{(3)}, \rho_{3}{ }^{(3)}\right) & =\left(\left(1-{\rho_{2}}^{2}\right) / \rho_{3}, \rho_{2},\left(1-{\rho_{2}}^{2}\right) / \rho_{1}\right) \equiv T\left(\rho_{1}, \rho_{2}, \rho_{3}\right), \\
\left(\rho_{1}{ }^{(4)}, \rho_{2}{ }^{(4)}, \rho_{3}{ }^{(4)}\right) & =S\left(T\left(\rho_{1}, \rho_{2}, \rho_{3}\right)\right) \\
\left(\rho_{1}{ }^{(5)}, \rho_{2}{ }^{(5)}, \rho_{3}{ }^{(5)}\right) & =T\left(S\left(\rho_{1}, \rho_{2}, \rho_{3}\right)\right) \\
\left(\rho_{1}{ }^{(6)}, \rho_{2}{ }^{\left({ }^{6}\right)}, \rho_{3}{ }^{(6)}\right) & =S\left(T\left(S\left(\rho_{1}, \rho_{2}, \rho_{3}\right)\right)\right) .
\end{aligned}
$$

If a domain $B$ in the $Z$-plane, $Z=X+i Y$, bounded by three closed (twice continuously differentiable) curves $\Gamma^{(p)}, \nu=1,2,3$, is given, one can normalize the mapping onto $\Omega$ requiring that $\Gamma_{1}, \Gamma_{3}$ and $\Gamma_{2}$ go into $\beta_{1}, \beta_{3}$ and $\beta_{2}$, respectively. By these requirements the mapping is normalized in a unique manner.

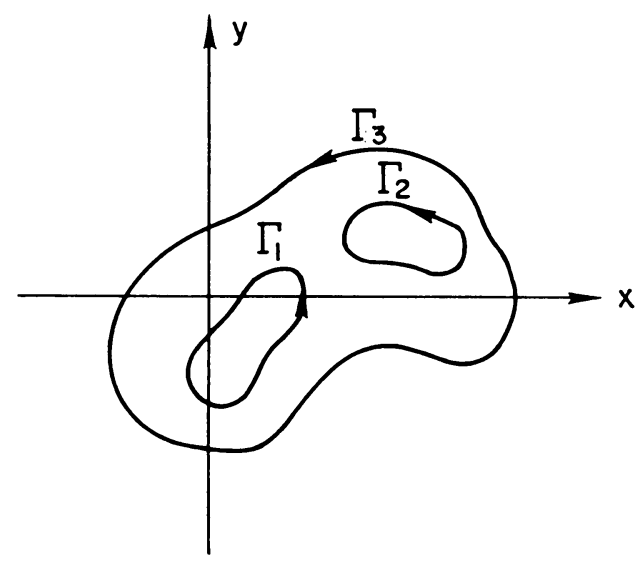

Figure 2. The domain $B$.

Let $\rho_{1}$ and $\rho_{3}$ be the inner and outer radii of the ring, respectively, and let $\rho_{2}$ be the radius of the circular hole and let $\beta_{1}, \beta_{2}, \beta_{3}$ be the three oriented circular boundary components (see Fig. 1).

In this section we shall discuss the construction of a set of orthogonal functions with a single-valued integral for the ring domain $\Omega$ with a circular hole. To obtain 
such a set, it suffices to orthogonalize the functions $z^{n}, n=0,1,2, \cdots ; z^{m},(1-z)^{m}$, $m=-2,-3,-4, \cdots$.

The first part of this procedure is the determination of the inner products of all of the above functions. Letting $C=\beta_{3}-\beta_{1}-\beta_{2}=\partial \Omega$ and using Green's identity, we have

$$
\begin{aligned}
\left(z^{m}, z^{k}\right) & =\iint_{B} z^{m} \bar{z}^{k} d \omega=\frac{1}{2 i} \int_{C} z^{m} \frac{\bar{z}^{k+1}}{k+1} d z \\
& =\frac{1}{2 i(k+1)}\left[\int_{\beta_{3}-\beta_{1}-\beta_{2}} z^{m \bar{z}^{k+1}} d z\right] \\
& =\frac{\pi}{(k+1)}\left[\delta_{m k}\left({\rho_{3}}^{2 k+2}-{\rho_{1}}^{2 k+2}\right)-\sum_{l=0}^{\infty}\left(\begin{array}{c}
m \\
l
\end{array}\right)\left(\begin{array}{c}
k+1 \\
l+1
\end{array}\right){\rho_{2}}^{2 l+2}\right] .
\end{aligned}
$$

Here and in the following

$$
\left(\begin{array}{l}
m \\
n
\end{array}\right)=0 \quad \text { if } \quad n<0, \quad\left(\begin{array}{l}
m \\
n
\end{array}\right)=1 \quad \text { if } \quad n=0
$$

and

$$
\left(\begin{array}{l}
m \\
n
\end{array}\right)=\frac{m(m-1) \cdots(m-n+1)}{n !} \text { if } n>0 .
$$

Hence if $m \geqq 0$ and $n>m$, then

$$
\left(\begin{array}{c}
m \\
n
\end{array}\right)=0 .
$$

Thus the infinite sum indicated above is actually a finite sum unless both $m$ and $k$ are negative.

Further we have

$$
\begin{aligned}
&\left((1-z)^{m},(1-z)^{k}\right)=\iint_{B}(1-z)^{m} \overline{(1-z)^{k}} d \omega=\frac{1}{2 i} \int_{C}(1-z)^{m}\left[-\frac{(1-\bar{z})^{k+1}}{k+1}\right] d z \\
&=-\frac{1}{2 i(k+1)}\left[\int_{\beta_{3}-\beta_{1}-\beta_{2}}(1-z)^{m}(1-\bar{z})^{k+1} d z\right] \\
&=\frac{\pi}{(k+1)}\left[\sum_{j=\max (0, k-m)}^{\infty}\left(\begin{array}{c}
m \\
m-k+j
\end{array}\right)\left(\begin{array}{c}
k+1 \\
j
\end{array}\right){\rho_{3}}^{2(k+1-j)}\right. \\
&\left.-\sum_{l=0}^{\infty}\left(\begin{array}{c}
m \\
l
\end{array}\right)\left(\begin{array}{c}
k+1 \\
l+1
\end{array}\right) \rho_{1}{ }^{2 l+2}-\delta_{m k} \rho_{2}{ }^{2 k+2}\right] .
\end{aligned}
$$

Finally we compute

$$
\begin{aligned}
\left((1-z)^{m}, z^{k}\right) & =\iint_{B}(1-z)^{m} \bar{z}^{k} d \omega=\frac{1}{2 i} \int_{C}(1-z)^{m} \frac{\bar{z}^{k+1}}{k+1} d z \\
& =\frac{1}{2 i(k+1)}\left[\int_{\beta_{3}-\beta_{1}-\beta_{2}}(1-z)^{m} \bar{z}^{k+1} d z\right] \\
& =\frac{\pi}{(k+1)}(-1)^{k}\left[\left(\begin{array}{c}
m \\
m-k
\end{array}\right) \rho_{3}{ }^{2 k+2}-\left(\begin{array}{c}
m \\
k
\end{array}\right) \rho_{1}{ }^{2 k+2}\right] .
\end{aligned}
$$

Note that 


$$
\left(\begin{array}{c}
m \\
n
\end{array}\right)=0 \text { if } n<0 \text {. }
$$

The next step in the construction of orthogonal functions is to order the functions $z^{n}, n=0,1,2, \cdots ; z^{m},(1-z)^{m}, m=-2,-3,-4, \cdots$, as follows: $1, z, z^{2}, z^{-2},(1-z)^{-2}, z^{3}, z^{-3},(1-z)^{-3}, \cdots \equiv \alpha_{1}, \alpha_{2}, \alpha_{3}, \cdots$ and then apply the Gram-Schmidt orthogonalization procedure to get $P_{n}(z)=\sum_{k=1}^{n} a_{n k} \alpha_{k}, n=1,2,3, \cdots$. The $a_{n k}$ are determined recursively by

$$
a_{n k}=c_{n k} /\left(\sum_{i=1}^{n} \sum_{j=1}^{n} c_{n i} c_{n j}\left(\alpha_{i}, \alpha_{j}\right)\right)^{1 / 2}
$$

where

$$
\begin{aligned}
c_{n k} & =-\sum_{i=k}^{n-1} a_{i k}\left(\sum_{j=1}^{i} a_{i j}\left(\alpha_{n}, \alpha_{j}\right)\right), \quad k=1,2, \cdots, n-1, \\
c_{n n} & =1 .
\end{aligned}
$$

The $\left\{P_{n}(z)\right\}, n=1,2, \cdots$, then form a complete set of orthonormal functions.

The invariant $J_{\Omega}$ is given by formula (1.2), where $K_{\Omega}(z, \bar{z})=\sum_{n=1}^{\infty} P_{n}(z) P_{n}(\bar{z})$ since all $a_{n k}$ are real (see Fig. 3, p. 533).

The orthogonalization of the set $Z^{n}, n=0,1,2, \cdots ; Z^{m},(1-Z)^{m}$, $m=-2,-3,-4, \cdots$, over $B$ proceeds in an analogous way (see also Section 6 ). In addition see [D-R. 1, 2].

4. The Construction of Coordinate Systems in $\Omega$ and $B$ Which Go Into Each Other by CT's. As we discussed in Section 2, the theory of the kernel function enables us to determine the invariant $J_{B}$. Using $J_{B}$, we shall map the given triplyconnected domain $B$ on a canonical domain $\Omega$, bounded by two concentric circles and a circular hole. As indicated in Section 2, we choose as the moduli the radii of the three boundary circumferences. We repeat that

$$
J_{\Omega}(z, \bar{z})=J_{B}(W(z), \quad \overline{W(z)}) 1 / 2
$$

and on the boundary $J_{\Omega}(z, \bar{z})$ assumes a constant value, namely $2 \pi$.

We denote segments of the level lines $J_{\Omega}(z, \bar{z})=$ const by $j$, the segments of orthogonal trajectories to $j$ by $p$.

At the critical points, say $z_{\kappa}$, of the invariant $J_{\Omega}(z, \bar{z})$ it holds

$$
\begin{aligned}
J_{\kappa} & =J_{\Omega}\left(z_{\kappa}, \bar{z}_{\kappa} ; \rho_{\mu}\right), \\
\left.\frac{\partial J_{\Omega}}{\partial z}\right|_{z=z_{\kappa} ; \bar{z}=\bar{z}_{\kappa}} & =0,\left.\quad \frac{\partial J_{\Omega}}{\partial \bar{z}}\right|_{z=z_{\kappa} ; \bar{z}=\bar{z}_{\kappa}}=0 .
\end{aligned}
$$

As we mentioned before, every triply-connected domain $B, \partial B=\Gamma_{1},+\Gamma_{2}+\Gamma_{3}$, can be mapped onto a canonical domain $\Omega$. Here $\Gamma_{\kappa}, \kappa=1,2,3$, are three closed curves, each of which is homeomorphic to a circle. $\Omega$ is a domain bounded by two concentric circles with the center at the origin and radius $\rho_{1}$ and $\rho_{3}, \rho_{3}>\rho_{1}$, and a third circle, with the center at $z=1$ and radius $\rho_{2}$.

Thus, if a domain $B$ is given, it is known that there exists a canonical domain $\Omega$, and the normalized moduli $\rho_{1}, \rho_{2}, \rho_{3}$ of $B$ are uniquely determined by $B$. In order 


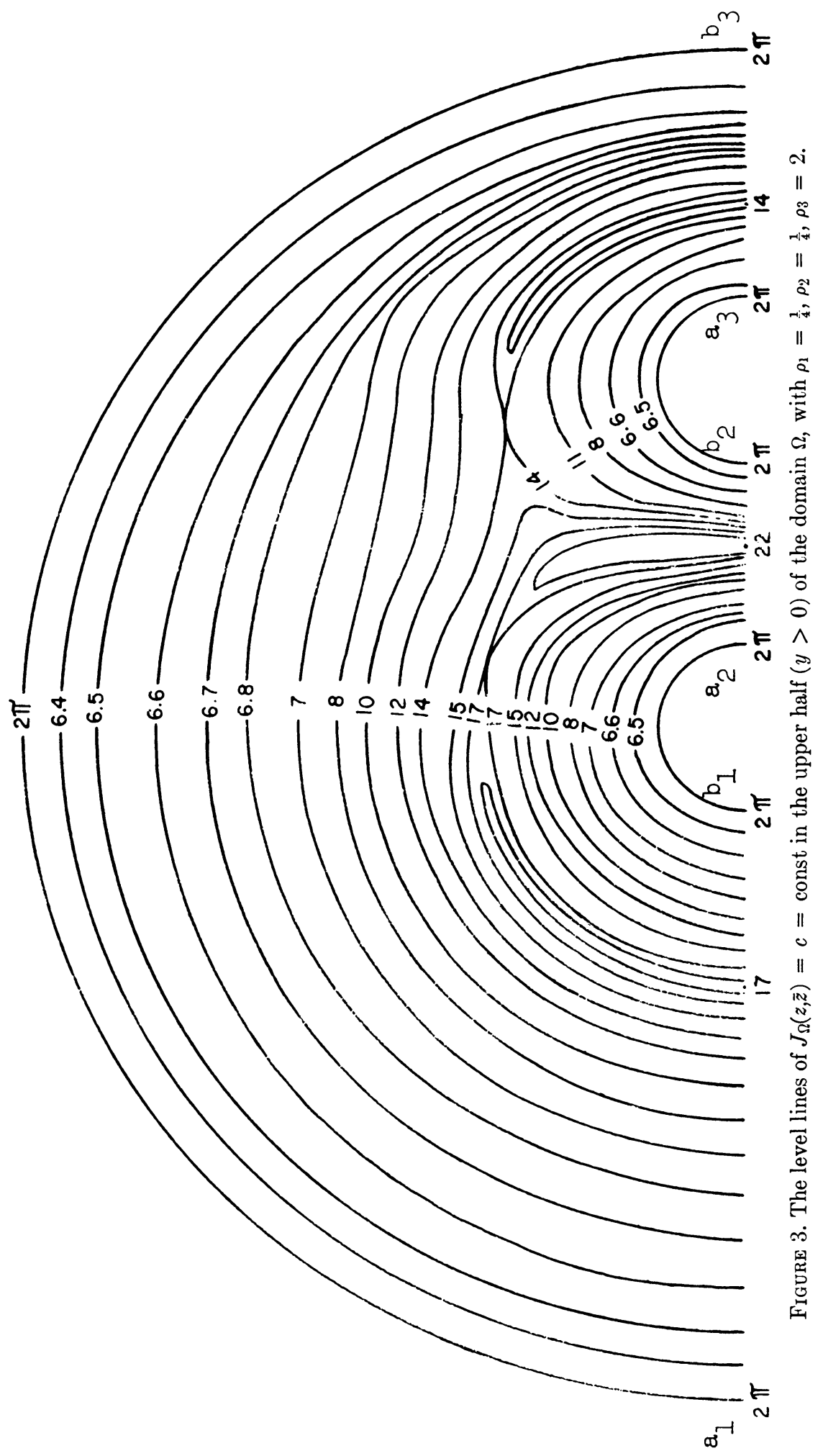


to apply our procedure, we make some hypotheses.

Let $M_{\kappa}$ be the largest maxima of $J_{\Omega}(z, \bar{z})$ on $\sigma\left(a_{k} b_{k}\right)$ with respect to the values of $J_{\Omega}(z, \bar{z})$ for $z \in \sigma\left(a_{k} b_{\kappa}\right) . \sigma\left(a_{k} b_{k}\right)$ means the segment of the $x$-axis with the endpoints $a_{k}, b_{k}$. See Fig. 3.

(a) We assume that in a certain segment $T$ (to be described later) of the threedimensional $M_{1}, M_{2}, M_{3}$-space, $M_{\kappa}\left(\rho_{1}, \rho_{2}, \rho_{3}\right)$ are continuously differentiable functions of $\rho_{1}, \rho_{2}, \rho_{3}$. Further we assume that $\partial\left(M_{1}, M_{2}, M_{3}\right) / \partial\left(\rho_{1}, \rho_{2}, \rho_{3}\right) \neq 0$ in $T$.

If $B$ in the $X, Y$-plane is not symmetric* with respect to the $X$-axis, we make the following additional assumption:

$\left(b_{1}\right)$ To every $\Gamma_{\kappa}, \kappa=1,2,3$, there exists one and only one pair of minimaxes $\mu_{\kappa}$ of $J_{B}$ at the points $Z_{\kappa \nu}, \nu=1,2$, possessing the following property: $Z_{\kappa 1}$ and $Z_{\kappa 2}$ can be connected by segments $s_{k 1}$ and $s_{\kappa 2}$ so that $s_{k 1} \cup s_{k 2}$ is homotopic to $\Gamma_{\kappa}$, and $s_{\kappa \nu}$ are segments of the level line $J_{B}(Z, \bar{Z})=J_{B}\left(Z_{\kappa \nu}, \bar{Z}_{\kappa \nu}\right)$. (We note that $J_{B}\left(Z_{\kappa 1}, \bar{Z}_{\kappa 1}\right)=J_{B}\left(Z_{\kappa 2}, \bar{Z}_{\kappa 2}\right)$.)

The second part of the assumption will be formulated in the following, see $\left(b_{2}\right)$.

Remark. A deeper mathematical investigation shows that a large part of the assumptions formulated as hypotheses $(a),\left(b_{1}\right)$ and $\left(b_{2}\right)$ can be rigorously proved. Since we do not want to become involved in too complicated considerations and since the numerical computation shows the validity of our assumption in all considered cases, we formulate the above additional assumptions as hypotheses.

Thus, suppose the domain $B, \partial B=\cup_{k=1}^{3} \Gamma_{k}$, is given. We determine the orthogonal functions $\Phi_{\nu}(Z)$, the invariant $J_{B}(Z, \bar{Z})$, its level lines ( $j$-lines) and their orthogonal trajectories ( $p$-lines). For details see the example (Section 6$)$.

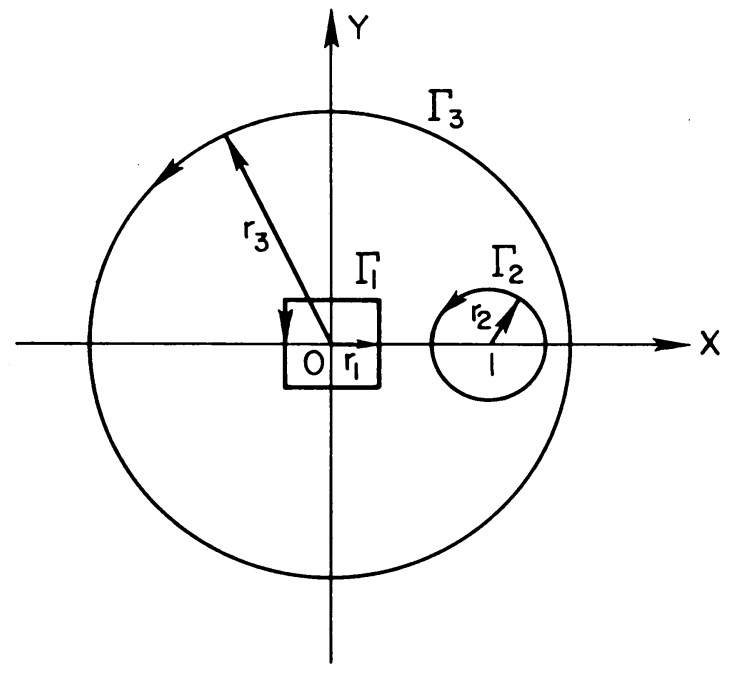

FIgURE 4. The domain $B$ of the example.

As the first problem we shall determine in $B$ the images $\sigma\left(A_{\kappa} B_{\kappa}\right), \kappa_{\kappa}=1,2,3$, of the segments of the $x$-axis. $\sigma\left(a_{k} b_{k}\right)$ are segments of lines which are perpendicular to the level lines $J_{\Omega}(z, \bar{z})=$ const. This property is preserved under conformal mapping, and therefore the segments $\sigma\left(A_{\kappa} B_{\kappa}\right)=W\left(\sigma\left(a_{\kappa} b_{\kappa}\right)\right)$ are segments of $p$-lines. If $B$ is symmetric with respect to the $X$-axis (for example, see Fig. 4, p. 534), then the

* If the boundary components of the domain $B$ are symmetric with respect to the $X$-axis, our considerations are greatly simplified. 
segments $W\left(\sigma\left(a_{\kappa} b_{\kappa}\right)\right)=\sigma\left(A_{\kappa} B_{\kappa}\right)$ are the three line segments determined by the intersection of $B$ with the $X$-axis. If, however, $B$ is not symmetric, then, in accordance with assumption $\left(b_{1}\right)$, there exist for $\Gamma_{\kappa}$ a pair of minimaxes, say at the points $Z_{\kappa \nu}$, $\nu=1,2$, and the segments $s_{\kappa \nu}$ of $j$-lines, which connect these points. In $\Omega$, the corresponding minimaxes at $z_{\kappa \nu}=W^{-1}\left(Z_{\kappa \nu}\right)$ must either lie on the $x$-axis or be symmetric with respect to the $x$-axis. The segments $s_{\kappa \nu}, \nu=1,2$, lie in $B$. Let $P_{\kappa \nu}$ be the points bisecting $s_{k \nu}$ (the distances being measured using the metric (1.1)). Then consider the two $p$-segments $p_{\kappa \nu}$ through the points $P_{\kappa \nu}, \nu=1,2$, and orthogonal to $s_{\kappa \nu}, \nu=1,2$. It is seen that the points $z_{\kappa \nu}=W^{-1}\left(Z_{\kappa \nu}\right), \nu=1,2$, lie on the $x$-axis if and only if at least one of the boundary components $\beta_{l}, l=1,2,3$, does not contain at least one of the endpoints of $p_{\kappa 1}$ or $p_{\kappa 2}$. Thus we make the hypothesis:

$\left(b_{2}\right)$ For every $\kappa=1,2,3$, each of the three boundary components $\beta_{l}, l=1,2,3$, contains at least one of the endpoints of $p_{\kappa 1}$ or $p_{\kappa 2}$. in" $B$.

Then it follows that the $p$-segments $p_{\kappa 1}$ are the images $W\left(\sigma\left(a_{k} b_{\kappa}\right)\right), \kappa=1,2,3$,

Now we proceed to the determination of moduli $\rho_{\kappa}, \kappa=1,2,3$, of $B$. For a discrete set of triples $\rho_{1}, \rho_{2}, \rho_{3}$ we have determined the corresponding triples of maxima $M_{1}, M_{2}, M_{3}$ of $J_{\Omega}(z, \bar{z})$ in Tables $1 \mathrm{~A}, 1 \mathrm{~B}, 1 \mathrm{C}$. Let the maxima of $J_{B}(Z, \bar{Z})$ on the segments $W\left(\sigma\left(a_{\kappa} b_{\kappa}\right)\right)$ be $M_{\kappa}, \kappa=1,2,3$. We determine four triples $M_{\kappa}{ }^{(\nu)}$, $\nu=1,2,3,4$, from our tables so that $\left\{M_{\kappa}\left({ }^{(0)}\right\}\right.$ is an interior point of the tetrahedron $T$, with vertices at the points $\left\{M_{1}{ }^{(\nu)}, M_{2}{ }^{(\nu)}, M_{3}{ }^{(\nu)}\right\}, \nu=1,2,3,4$. We choose $T$ to be the domain mentioned in hypothesis (a), p. 534 .

Thus by a well-known result, see [O.1, Theorem 25.1 and Section 26], the values $\left\{\rho_{\kappa}{ }^{(0)}\right\}$ corresponding to $\left\{M_{\kappa}{ }^{(0)}\right\}$ can be computed by the Newton-Raphson method.

We determine the level lines $j_{\Omega}=\left[J_{\Omega}(z, \bar{z}) \equiv J_{\Omega}\left(z, \bar{z} ; \rho_{1}{ }^{(0)}, \rho_{2}{ }^{(0)}, \rho_{3}{ }^{(0)}\right)=\right.$ const $]$ and their orthogonal trajectories $p_{\Omega}=W^{-1}\left(p_{B}\right)$. We choose the trajectories $p_{\Omega}$ so that the (noneuclidean) distance of the line $p_{\Omega}=W^{-1}\left(p_{B}\right)$ from the segments $\sigma\left(a_{\kappa} b_{\kappa}\right)$ of the $x$-axis (or from the minimaxes $z_{\kappa_{\nu}}$ ) is the same as the (noneuclidean) distance between $p_{B}$ and $W\left(\sigma\left(a_{\kappa} b_{k}\right)\right)$ (or from $\left.Z_{\kappa \nu}=W\left(z_{\kappa \nu}\right)\right)$. Then the (curvilinear)

TABLE 1A

Tables of maximum triples $M_{1}, M_{2}, M_{3}$ corresponding to moduli $\rho_{1}, \rho_{2}, \rho_{3}$

\begin{tabular}{|c|c|c|c|c|}
\hline & & $3=1$. & & \\
\hline$\rho_{1}$ & $1 / 8$ & $1 / 4$ & $3 / 8$ & $1 / 2$ \\
\hline $1 / 8$ & $\begin{array}{l}30.0 \\
25.0 \\
15.8\end{array}$ & $\begin{array}{r}28.5 \\
23.9 \\
8.5\end{array}$ & $\begin{array}{r}23.0 \\
22.0 \\
6.4\end{array}$ & $\begin{array}{c}17.5 \\
18.7 \\
2 \pi\end{array}$ \\
\hline $1 / 4$ & $\begin{array}{l}25.2 \\
15.3 \\
15.5\end{array}$ & $\begin{array}{r}17.5 \\
14.8 \\
8.4\end{array}$ & $\begin{array}{r}13.0 \\
13.8 \\
6.4\end{array}$ & $\begin{array}{r}8.8 \\
12.0 \\
2 \pi\end{array}$ \\
\hline $3 / 8$ & $\begin{array}{l}21.4 \\
11.6 \\
15.3\end{array}$ & $\begin{array}{r}13.0 \\
11.3 \\
8.2\end{array}$ & $\begin{array}{r}7.8 \\
10.6 \\
6.3\end{array}$ & $\begin{array}{l}6.3 \\
9.1 \\
2 \pi\end{array}$ \\
\hline $1 / 2$ & $\begin{array}{r}16.8 \\
9.3 \\
14.7\end{array}$ & $\begin{array}{l}8.6 \\
9.0 \\
8.0\end{array}$ & $\begin{array}{l}6.3 \\
8.4 \\
6.3\end{array}$ & $\begin{array}{l}2 \pi \\
6.8 \\
2 \pi\end{array}$ \\
\hline
\end{tabular}




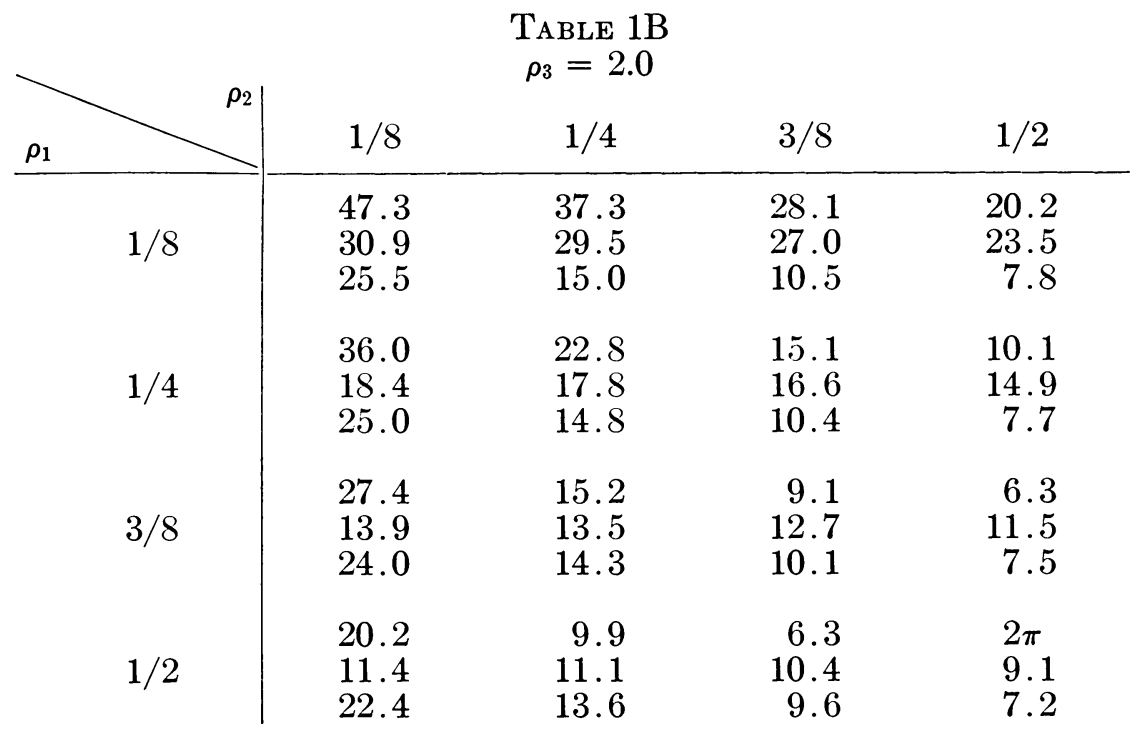

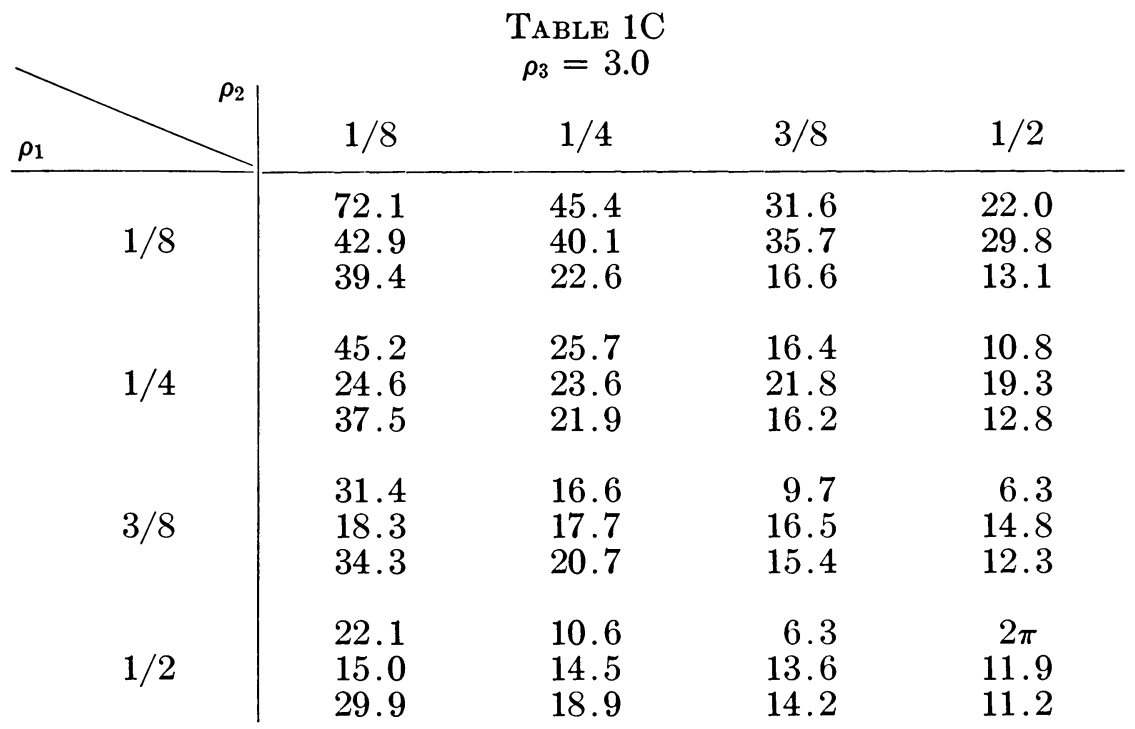

coordinate system $\left(j_{\Omega}, p_{\Omega}\right)$ goes by the CT $W$ into the system $\left(j_{B}, p_{B}\right)$.

5. A Remark About Another Method for Construction of a CT, Mapping a Domain $B$ onto $G$. Making several additional assumptions in Section 4 (which have not been proved), we described a procedure how to determine the image (in $B$ ) of a segment of the $x$-axis (in $\Omega$ ). Using this result, we then determine the function $Z=w(z)$, mapping $\Omega$ onto $B$.

It should be noted that, making much weaker assumptions and using deeper mathematical results, one can determine the mapping functions $w(z)$. The method requires procedures which are constructive, but which are from the numerical point of view more difficult than those used in Section 4. 
In the following we shall indicate the basic idea of this (numerically more difficult) procedure.

(1) We assume that there exists at least one maximum set of $J_{B}(z, \bar{z})$ which is a point and lies in $B$. Suppose that among the maximum points there exists only one point $z=t$ for which $J_{B}(t, \bar{t})=J_{0}$ and at all other maxima points $J_{B}(z, \bar{z}) \neq J_{0}$. If two triply-connected domains, say $B$ and $G$, can be mapped onto each other and each of them has one point maximum at $t$ and $T$, respectively, then $J_{B}(t, \bar{t})=$ $J_{G}(T, \bar{T})$. In this way we obtain a necessary condition in order that $B$ can be mapped onto $G$. To obtain a sufficient condition and the desired mapping function, we determine the representative domains $\hat{R}(B, t)$ and $\hat{R}(G, T)$ (see [B.4, p. $27 \mathrm{ff}$.]). In accordance with results of [B.4, pp. 31, 32], two representative domains $\hat{R}(B, t)$ and $\hat{R}(G, T)$ can be mapped onto each other only by a linear transformation

$$
W=a_{1} w+a_{2},
$$

where $a_{k}$ are complex constants, i.e., by translation, rotation and dilatation around the center. Thus if the domains $\hat{R}(B, t)$ and $\hat{R}(G, T)$ are constructed, one can recognize whether a mapping of the form (1) maps the domain $\hat{R}(B, t)$ onto $\hat{R}(G, T)$.

6. An Example of an Application of the Procedure of Sections 3 and 4. Using the results of the previous sections, we now determine the CT of a domain $B$ onto a canonical domain $\Omega$. We take for $B$ the symmetric domain bounded by a circle of radius $r_{3}$ and a square of width $2 r_{1}$ centered at $z=0$, and the circle of radius $r_{2}$ centered at $z=1$. Here $r_{3}>r_{1}+2 r_{2}$, and the axis of symmetry lies on the $x$-axis (see Fig. 4).

First we compute a complete set of orthogonal functions for $B$, from which we determine the kernel function $K_{B}$ and the invariant $J_{B}$ of $B$. Then we choose the largest maxima $M_{\nu}=J_{B}\left(z_{\nu}, \bar{z}_{\nu}\right)$ at the points $Z_{\nu}$ located on the segments $\left(A_{\nu}, B_{\nu}\right)$ of the $x$-axis. Thus, using the procedure described above, we determine the moduli of $B$. Letting $\Omega$ denote the canonical domain described above with these moduli, and using $J_{B}, J_{\Omega}, d s_{B}$, and $d s_{\Omega}$, we can then construct the CT from $B$ onto $\Omega$.

First we shall discuss the construction of a set of orthogonal functions with a single-valued integral for the domain $B$. It suffices to orthogonalize the functions $Z^{n}, n=0,1,2, \cdots ; Z^{m},(1-Z)^{m}, m=-2,-3,-4, \cdots$ Letting $C=\Gamma_{3}-\Gamma_{1}-\Gamma_{2}=\partial B$ (see Fig. 4) and, using Green's identity, we have

where

$$
\begin{aligned}
\left(Z^{m}, Z^{k}\right) & =\iint_{B} Z^{m} \bar{Z}^{k} d \omega=\frac{1}{2 i} \int_{C} Z^{m} \frac{\bar{Z}^{k+1}}{k+1} d Z=\frac{1}{2 i(k+1)}\left[\int_{\Gamma_{3}-\Gamma_{1}-\Gamma_{2}} Z^{m} \bar{Z}^{k+1} d Z\right] \\
& =\frac{\pi}{(k+1)}\left[\delta_{m k} r_{3}{ }^{2 k+2}-\sum_{l=0}^{\infty}\left(\begin{array}{c}
m \\
l
\end{array}\right)\left(\begin{array}{c}
k+1 \\
l+1
\end{array}\right) r_{2}{ }^{2 l+2}\right]+a_{m k},
\end{aligned}
$$

$$
\begin{aligned}
& a_{m k}=\left\{\frac{(-1)^{k+1}}{k+1}(\sqrt{ } 2)^{2 k+m+5} r_{1}{ }^{m+k+2} \sin \left(\frac{m+k+1}{2} \pi\right) \sum_{l=0}^{\infty}\left(\begin{array}{c}
k+1 \\
l
\end{array}\right)\right. \\
&\left.\times(-\sqrt{ } 2)^{-l} \frac{\sin ((m+l+1) \pi / 4)}{m+l+1}\right\} \\
& \times\left[1+\cos (m-k) \frac{\pi}{2}+i \sin (m-k) \frac{\pi}{2}\right] .
\end{aligned}
$$




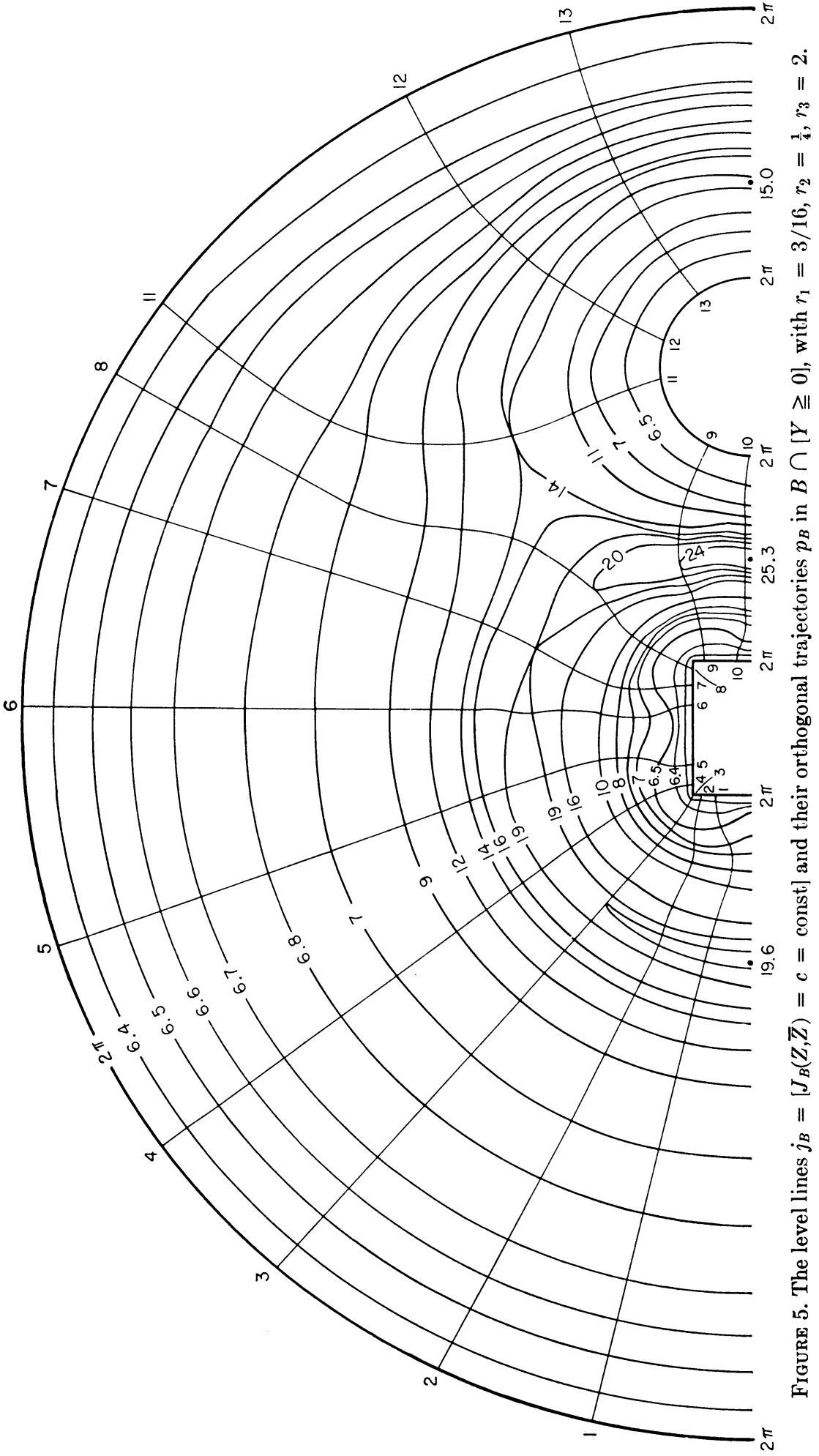




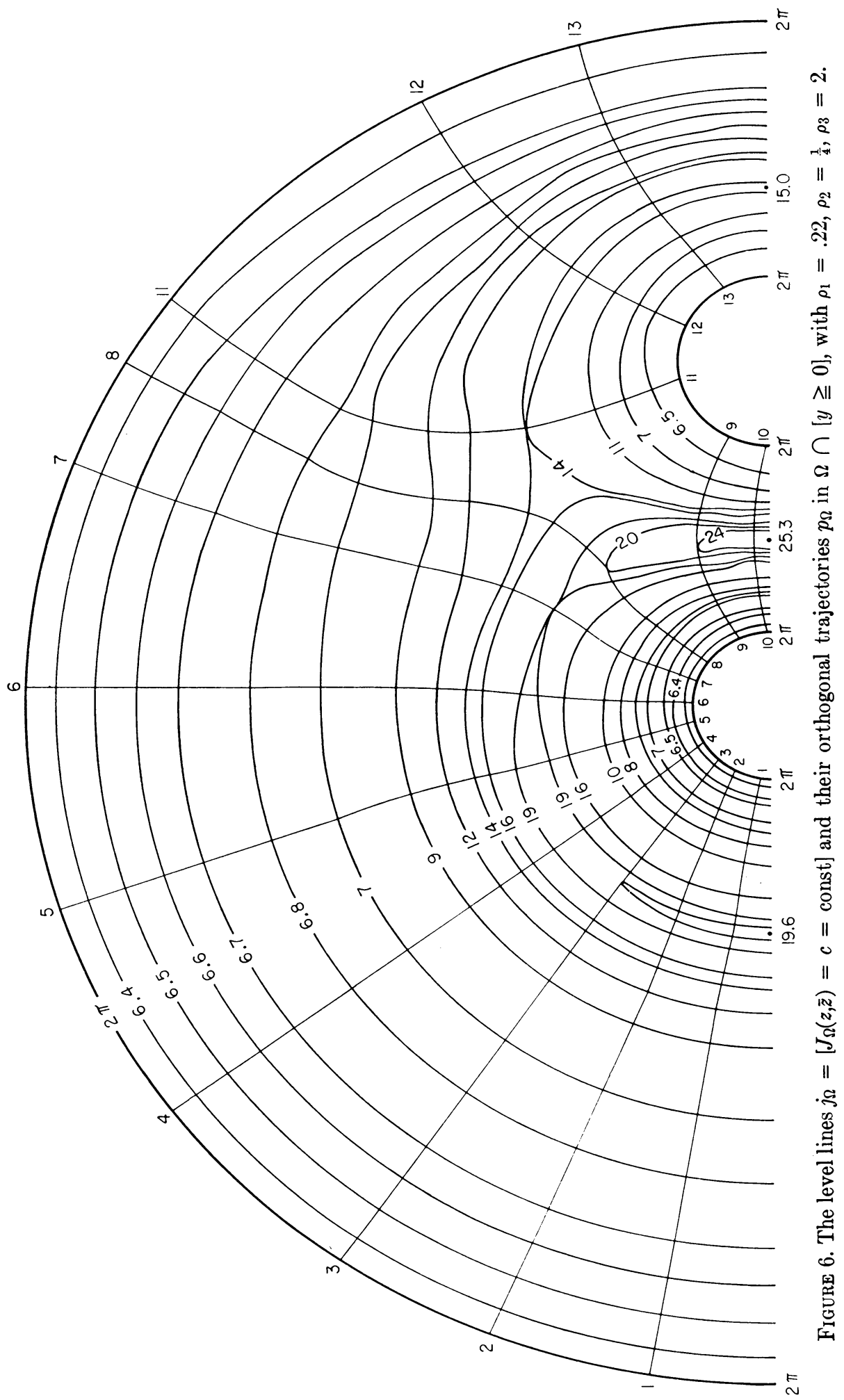


We now determine

$$
\begin{aligned}
\left((1-Z)^{m}, Z^{k}\right) & =\frac{1}{2 i} \int_{C}(1-Z)^{m} \frac{\bar{Z}^{k+1}}{k+1} d Z=\frac{1}{2 i(k+1)}\left[\int_{\Gamma_{3}-\Gamma_{1}-\Gamma_{2}}(1-Z)^{m} \bar{Z}^{k+1} d Z\right] \\
& =\frac{\pi}{k+1}(-1)^{k}\left(\begin{array}{c}
m \\
m-k
\end{array}\right) r_{3}{ }^{2 k+2}+b_{m k},
\end{aligned}
$$

where

$$
b_{m k}=\sum_{j=0}^{\infty}\left(\begin{array}{c}
m \\
j
\end{array}\right)(-1)^{j} a_{j k}, \quad \text { if } \quad r_{1}<\frac{1}{\sqrt{ } 2} .
$$

Similarly, for the determination of $\left((1-Z)^{m},(1-Z)^{k}\right)$ we have

$$
\begin{aligned}
& \left((1-Z)^{m},(1-Z)^{k}\right)=-\frac{1}{2 i(k+1)}\left[\int_{\Gamma_{3}-\Gamma_{1}-\Gamma_{2}}(1-Z)^{m}(1-\bar{Z})^{k+1} d Z\right] \\
& =\frac{\pi}{k+1}\left[\sum_{j=\max (0, k-m)}^{\infty}\left(\begin{array}{c}
m \\
m-k+j
\end{array}\right)\left(\begin{array}{c}
k+1 \\
j
\end{array}\right) \rho_{3}{ }^{2(k+1-j)}\right. \\
& \left.-\delta_{m k} \rho_{2}{ }^{2 k+2}\right]+c_{m k},
\end{aligned}
$$

where

$$
c_{m k}=\sum_{r=0}^{\infty}(-1)^{r}\left[\sum_{l=0}^{r}\left(\begin{array}{c}
m \\
l
\end{array}\right)\left(\begin{array}{c}
k+1 \\
r-1
\end{array}\right) a_{l(r-l-1)}\right], \quad \text { if } \quad r_{1}<\frac{1}{\sqrt{ } 2} .
$$

In order to construct orthogonal functions, we order the functions $Z^{n}$, $n=0,1,2, \cdots ; Z^{m},(1-Z)^{m}=-2,-3,-4, \cdots$ as follows: $1, Z, Z^{2}, Z^{-2}$, $(1-Z)^{-2}, Z^{3}, Z^{-3},(1-Z)^{-3}, \cdots \equiv \alpha_{1}, \alpha_{2}, \alpha_{3}, \cdots$ and then apply the GramSchmidt procedure to get

$$
P_{n}(Z)=\sum_{k=1}^{n} a_{n k} \alpha_{k}, \quad n=1,2,3, \cdots .
$$

The $a_{n k}$ are determined recursively by

where

$$
a_{n k}=c_{n k} /\left(\sum_{i=1}^{n} \sum_{j=1}^{n} c_{n i} \bar{c}_{n j}\left(\alpha_{i}, \alpha_{j}\right)\right)^{1 / 2},
$$

$$
\begin{aligned}
c_{n k} & =-\sum_{i=k}^{n-1} a_{i k}\left(\sum_{j=1}^{i} \bar{a}_{i j}\left(\alpha_{n}, \alpha_{j}\right)\right), \quad k=1,2, \cdots, n-1 \\
c_{n n} & =1 .
\end{aligned}
$$

The $\left\{P_{n}(Z)\right\}, n=1,2, \cdots$, then form a complete set of orthonormal functions.

The invariant $J_{B}$ is thus given by the formula (1.2), namely,

$$
J_{B}(Z, \bar{Z})=\left|\begin{array}{ll}
K & K_{\bar{Z}} \\
K_{Z} & K_{Z \bar{Z}}
\end{array}\right| / K^{3}
$$

and the invariant line element is given by $d s_{B}{ }^{2}=K_{B}|d Z|^{2}$, where

$$
K_{B} \equiv K_{B}(Z, \bar{Z})=\sum_{n=1}^{\infty} P_{n}(Z) \overline{P_{n}(Z)} .
$$


We now choose the particular values $r_{1}=3 / 16, r_{2}=1 / 4$ and $r_{3}=2$ for the domain $B$. Evaluating $J_{B}$ according to the above formulae, we determine that the desired triple of maxima $\left(M_{1}, M_{2}, M_{3}\right)$ is in this case

$$
\left(M_{1}, M_{2}, M_{3}\right) \equiv(25.3,19.6,15.0) \text {. }
$$

Then, by interpolation of the values in the tables at the end of Section 4 and according to the method discussed there, we obtain as the triple of moduli for $B$

$$
\left(\rho_{1}, \rho_{2}, \rho_{3}\right) \equiv\left(.22, \frac{1}{4}, 2\right) .
$$

Thus $B$ can be mapped onto the canonical domain $\Omega$ of circular radii $\rho_{1}=.22$, $\rho_{2}=\frac{1}{4}, \rho_{3}=2$. See Fig. 1 . We choose in both cases the point where $J_{B}=J_{\Omega}=25.3$ as the origin.

We now determine the CT $W^{-1}$ from $B$ onto $\Omega$. For this purpose we compute the level lines of $J_{B}$ and $J_{\Omega}$, see Figs. 5 and 6 . Some representative level lines of $J_{B}$ are depicted in Fig. 5 and the corresponding curves for $J_{\Omega}$ are shown in Fig. 6. (In each case these level lines $j$ are the curves intersecting the $x$-axis orthogonally.)

The next step in the construction of the CT $W$ is the determination of some representative $p$-curves orthogonal to the level lines of $J_{B}$ and $J_{\Omega}$. These curves are selected by measuring prescribed invariant lengths along certain of the level lines $j$ by the metric (1.1) and forming the curves orthogonal to these level lines at the prescribed points. Thus with the aid of the $d s_{B}$ line element we determine some representative "orthogonal" curves for $B$, as shown in Fig. 5; and in Fig. 6 are depicted the corresponding "orthogonal" curves for $\Omega$ obtained by use of the $d s_{\Omega}$ line element. In each case these "orthogonal" curves are those intersecting the boundaries of $B$ and $\Omega$. In such a way we obtain two systems of intersecting curves, one for $B$ and one for $\Omega$, and the correspondence of the points in these two frameworks represents the desired CT $W$.

Department of Mathematics

Stanford University

Stanford, California

B.1. S. Bergman, "Ueber die Existenz von Repräsentantenbereichen," Math. Ann., v. 102, 1929, pp. 430-446.

B.2. S. Bergman, "Ueber die Kernfunktion eines Bereiches und ihr Verhalten am Rande," J. Reine Angew. Math., v. 169, 1933, pp. 1-42, 172; 1934, pp. 89-128.

B.3. S. Bergman, Sur les Fonctions Orthogonales de Plusieurs Variables Complexes avec les Applications à la Théorie des Fonctions Analytiques, Interscience, New York, 1941; Memor. Sci. Math., No. 106, Gauthier-Villars, Paris, 1947. MR 2, 359; MR 11, 344.

B.4. S. Bergman, Sur la Fonction-Noyau d'un Domaine et ses Applications dans la Théorie des Transformations Pseudoconformes, Memor. Sci. Math., No. 108, Gauthier-Villars, Paris, 1948. MR 11, 344.

B.5. S. Bergman, The Kernel Function and Conformal Mapping, Math. Surveys, No. 5, Amer. Math. Soc., Providence, R. I., 1950. MR 12, 402.

B.6. S. Bergman, "Distinguished sets of invariants in the theory of pseudo-conformal transformations," J. Analyse Math., v. 13, 1964, pp. 317-353. MR 31 \#5987.

B.7. S. Bergman, "On boundary behavior of some domain functionals in the theory of functions of two complex variables," Bull. Soc. Sci. Lettres Eódź, v. 14, 1966, pp. 1-15. 
B.8. S. Bergman, "Some properties of pseudo-conformal images of circular domains in the theory of two complex variables," Proc. Conf. Complex Analysis (Minneapolis 1964), SpringerVerlag, Berlin, 1965, pp. 30-40. MR 32 \#2612.

B.9. S. Bergman, "Interior distinguished points in the theory of functions of two complex variables," J. Analyse Math., v. 18, 1967, pp. 7-21.

B-S. 1. S. Bergman \& M. Schiffer, "Kernel functions and conformal mapping," Compositio Math., v. 8, 1951, pp. 205-249. MR 10, 42.

D-R.1. P. Davis \& P. Rabinowitz, "A multiple purpose orthonormalizing code and its uses," J. Assoc. Comput. Mach., v. 1, 1954, pp. 183-191. MR 16, 751.

D-R.2. P. Davis \& P. Rabinowitz, "Numerical experiments in potential theory using orthonormal functions," J. Washington Acad. Sci., v. 46, 1956, pp. 12-17. MR 19, 686.

E.1. B. Epstein, Orthogonal Families of Analytic Functions, Macmillan, New York; CollierMacmillan, London, 1965. MR 31 \#4916.

F.1. B. A. Fuks, Special Chapters in the Theory of Analytic Functions of Several Complex Variables, Fizmatgiz, Moscow, 1963; English transl., Transl. Math. Monographs, Vol. 14, Amer. Math. Soc., Providence, R. I. MR 30 \#4979.

H.1. K. T. HAHN, "Minimum problems of Plateau type in the Bergman metric space," Pacific J. Math., v. 14, 1964, pp. 943-955. MR 29 \#6402.

K.1. L. V. Kantorovich, "Functional analysis and applied mathematics," Uspehi Mat. Nauk, v. 3, 1948, no. 6 (28), pp. 89-158; English transl., N.B.S. Report 1509, U. S. Dept. of Commerce, National Bureau of Standards, Los Angeles, Calif., 1952. MR 10, 380; MR 14, 766.

M.1. H. Meschkowskr, Hilbertsche Räume mit Kernfunktion, Die Grundlehren der mathematischen Wissenschaften, Band 113, Springer-Verlag, Berlin, 1962. MR 25 \#4326.

O.1. A. Ostrowski, Solution of Equations and Systems of Equations, Pure and Appl. Math., Vol. IX, Academic Press, New York, 1960. MR 23 \#B571.

T.1. L. Tonelli, Serie Trigonometriche, Bologna, 1928.

Z.1. K. ZARANKIEwicz, "Ueber ein numerisches Verfahren zur konformen Abbildung zweifach zusammenhängender Gebiete," Z. Angew. Math. Mech., v. 14, 1934, pp. 97-104. 